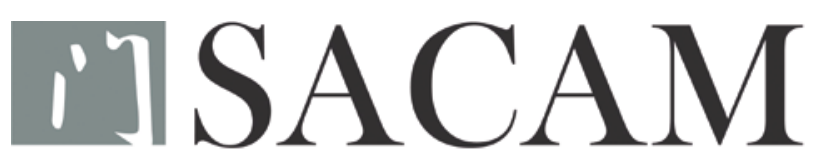

SCHWEIZERISCHE ÄRZTEGESELLSCHAFT FÜR

AKUPUNKTUR - CHINESISCHE MEDIZIN - AURIKULOMEDIZIN

\title{
KONGRESSVORSCHAU FÜR DIE SCHWEIZ
}

\section{KURSE DER SCHWEIZERISCHEN ÄRZTEGESELLSCHAFT \\ FÜR AKUPUNKTUR - CHINESISCHE MEDIZIN - AURIKULOMEDIZIN}

17.09.2015-28.05.2016

19.09.2015-28.05.2016

23.10.2015-18.06.2016

$06.11 .2015-14.11 .2015$

$22.02 .2016-26.02 .2016$

11.03.2016-19.03.2016

27.05.2016-10.06.2016
Unikurs Grundlagen Akupunktur-TCM

Unikurs TCM-Körperakupunktur I

Unikurs RAC-kontrollierte Akupunktur 1

Ohrakupunktur: Stufe 1-3

Unikurs RAC-kontrollierte Akupunktur 2 Stufe 5

Expertenkurs Davos für Ohrakupunktur/ Aurikulomedizin der 5. und 6. Stufe und klassische Akupunktur

Unikurs RAC-kontrollierte Akupunktur 2 Stufe 6

Unikurs RAC-kontrollierte Akupunktur 2 Prüfungsvorbereitung
Bern

Bern

Bern/Biel

Bern/Biel

Davos

Bern/Biel

Bern

(Terminänderungen vorbehalten)

\section{Weitere Informationen entnehmen Sie bitte den Ausschreibungen auf der Homepage} der SACAM: www.sacam.ch - Info Arzt - Fortbildung

Geschäftstselle:

SACAM - Schweizerischen Ärztegesellschaft für

Akupunktur - Chinesische Medizin - Aurikulomedizin

Postfach 2003, CH-8021 Zürich

Tel.: +41 844/200 200, Fax: +41 31/3324112

E-Mail:info@sacam.ch,Internet:www.sacam.ch 